\section{Attitudes towards the implementation of the Mental Health Act 2001}

In 2001 the Department of Health and Children published the Mental Health Act 2001, which comprised new legislation intended to replace the existing Mental Treatment Act of 1945.' A brief summary of the Mental Health Act 2001 is provided by O'Shea. ${ }^{2}$ Since 2001 , certain aspects of the Mental Health Act 2001 (such as the appointment of the Mental Health Commission) have been implemented, while other aspects (such as the appointment of Mental Health Tribunals) are in the process of being implemented. ${ }^{3}$

Amongst psychiatrists, positive aspects of the Act (such as automatic reviews of detention and revised procedures for involuntary admission) have been broadly praised, but concern has been expressed about other aspects, including the overall resource implications within the mental health sector. ${ }^{4.7}$ In this survey, we aimed to ascertain the views of psychiatrists in Ireland about the implementation of the Mental Health Act 2001, with a view to collecting data that would help inform a collaborative approach to the implementation of the new legislation.

\section{Methods}

Data collection

We designed a seven-page, self-administered, postal questionnaire. Firstly, we asked respondents to detail their 'grade' (registrar, senior registrar, consultant, retired consultant). The remainder of the questions explored respondents' views about the adequacy of training in relation to the Mental Health Act 2001, the adequacy of information available to stakeholders, and the likely effect of the new legislation on workloads and relationships with patients and teammembers. We also sought respondents' views on the overall feasibility of introducing the new legislation at current levels of resourcing. For most questions, we used ten-point scales for respondents to rate the strength of their responses. For example, question 1 read as follows: "Is your training in the new Act adequate, under the following headings? Score 1 for inadequate, 5 for adequate, and 10 for excellent." For this question, we provided a 'tick-box' grid in which respondents could rate the adequacy of their training (between 1 and 10) under four different headings (quality, quantity, geographical accessibility, and time accessibility).

Sample

Questionnaires were posted by the Irish College of Psychiatrists to all 535 psychiatrists (registrars, senior registrars, consultants and retired consultants) whose names and addresses appeared on the list kept by the Irish College of Psychiatrists in early 2004; this list includes all persons who are members of the Royal College of Psychiatrists (MRCPsych) and are recorded on the mailing list of the Irish College of Psychiatrists as residing in the Republic of Ireland. Data analysis

The Statistical Package for the Social Sciences (SPSS) was used for the analysis of data. ${ }^{8}$ Response data for all variables were non-normally distributed, so medians and modal values are reported. Non-parametric statistical tests were used for comparisons across grades: Mann-Whitney test for quantitative variables and Pearson's Chi square test for categorical variables (with Bonferroni-corrected levels of significance where appropriate, owing to multiple testing). In order to increase statistical power in comparisons across grades, the variable 'grade' was recoded into binary form: for such comparisons, the grade 'consultants' included practicing consultants and retired consultants (total $n=108$ ) and the grade 'junior doctors' included registrars and senior registrars (total $n=29$ ).

\section{Results}

Responses were received from 139 psychiatrists, yielding an overall response rate of $26.0 \%$. Of the 139 respondents, $18(12.9 \%)$ were registrars in psychiatry, 11 (7.9\%) were senior registrars in psychiatry, 103 (74.1\%) were consultant psychiatrists in active clinical practice, 5 (3.6\%) were retired consultant psychiatrists, and two respondents (1.4\%) did not provide information about their grade.

Respondents were asked to rate their training in relation to the Mental Health Act 2001 on a scale between 1 and 10 (with 1 indicating inadequate training; 5 indicating adequate training; and 10 indicating excellent training). Overall, 76.9\% of respondents rated the quality of training as less-thanadequate (score ${ }^{2} 4$ ); $81.5 \%$ rated the quantity of training as less-than-adequate; $76.4 \%$ rated the geographical accessibility of training as less-than-adequate; and $83.5 \%$ rated the time accessibility of training as less-than-adequate. A majority of respondents believed that the training needs of other mental health workers were not being met, indicating that less-than-adequate training (score ${ }^{2} 4$ ) was available to junior doctors $(89.4 \%)$, nursing staff $(87.3 \%)$, other members of the clinical team (89.4\%), secretarial staff (94.5\%) and administrative/managerial staff (85.6\%). Respondents also indicated that the information needs of other stake-holders in the mental health services were not being met, with over $90 \%$ of respondents expressing the view that less-than-adequate information had been provided to patients, carers, social workers, general practitioners, local authorities and voluntary agencies.

The majority of respondents (57.8\%) envisaged significant problems converting patients already detained under existing legislation to equivalents under the Mental Health Act 2001; a greater proportion of junior doctors $(81.5 \%)$ than consultants $(50.6 \%)$ envisaged problems in this area (Pearson Chi square 8.07; exact $p=0.007$ ). A majority of respondents felt the introduction of the Mental Health Act 2001 will result in an increase in workloads for consultant psychiatrists (93.0\%), junior doctors $(92.3 \%)$, community psychiatric nurses $(89.2 \%)$ and social workers $(82.5 \%)$. A majority of respondents (over $80 \%$ ) also envisaged that the introduction of the Mental Health Act 2001 will decrease resources available for other areas of the mental health services, including monetary resources, meeting/conference rooms, secretarial time, advocacy services for voluntary patients, medical records support and the availability of second opinions from colleagues.

Respondents also foresaw significant changes in their 
roles, with $60.0 \%$ expecting the Mental Health Act 2001 to result in decreased levels of patient contact and $71.6 \%$ expecting decreased levels of contact with outpatients in the community. Sixty per-cent of respondents expect increased levels of supervisory and management activity, with a greater proportion of junior doctors (83.3\%) than consultants $(53.6 \%)$ expecting increased activity in this area (Pearson Chi Square $=6.90$; exact $p=0.009$ ). In addition, $51.6 \%$ of respondents believe their relationships with patients will change following the introduction of the new legislation.

Finally, respondents were asked: "Overall, do you consider that implementation of the new legislation is feasible at present levels of service resourcing? Score 1 for completely infeasible; 5 for moderately or partially feasible; and 10 for completely feasible." The median score for this question was 3 and the modal score was 2 (range: 1-10). Overall, $80 \%$ of respondents felt it was less than 'moderately feasible' (score ?4) to implement the new legislation at current levels of resourcing and only two respondents (1.6\%) believed it to be completely feasible (score $=10$ ) at current resource levels (see Table).

\section{Discussion}

We found that a majority of psychiatrists who responded to this survey (in early 2004) believed that the introduction of the Mental Health Act 2001 will (a) have significant impact on resources in other areas of mental health services; (b) significantly change their relationships with patients and with colleagues; and (c) result in decreased levels of patient contact and increased levels of supervisory and management activity. Overall, $80 \%$ of respondents felt it was less than 'moderately feasible' to implement the new legislation at current levels of resources.

The central limitation of this study is the low response rate (26.0\%). This low response rate may be attributable to the length of the questionnaire ( 7 pages), the design of the questionnaire, or the views of respondents in relation to the subject matter. Though not unusual for a survey of this nature, this low response rate raises the possibility of response bias. It is difficult to hypothesise exactly how such a bias might have affected results: on the one hand, it could be hypothesized that psychiatrists who had strong negative feelings about the implementation of the new legislation were more likely to respond to the survey (thus biasing results in a 'negative' direction); though on the other hand it could be argued that psychiatrists with such negative feelings would be more likely not to respond to any further material they receive in the post relating to the Mental Health Act 2001 (thus biasing results in a 'positive' direction).

Finally, it is possible that there was a degree of response bias in both 'negative' and 'positive' directions and that these simply cancelled each other out. In the absence of any further information about the respondents, however, the possibility of significant bias remains, and this is a significant limitation.

This survey highlights the importance of providing clear information in relation to the implementation of the Mental Health Act 2001 to all stake-holders in mental health services. Such information is currently available from a range of sources. The website of the Mental Health Commission (www.mhcirl.ie), in particular, offers a wide range of excel-
Table 1: Psychiatrists' views on the feasibility of introducing the Mental Health Act 2001 at present levels of resourcing*

$\begin{array}{cccc}\text { Score (out of 10) } & n & \text { \% (out of 125) } & \text { Cumulative \% } \\ 1 & 37 & 29.6 & 29.6 \\ 2 & 25 & 20.0 & 49.6 \\ 3 & 31 & 24.8 & 74.4 \\ 4 & 7 & 5.6 & 80.0 \\ 5 & 18 & 14.4 & 94.4 \\ 6 & 0 & 0.0 & 94.4 \\ 7 & 2 & 1.6 & 96.0 \\ 8 & 1 & 0.8 & 96.8 \\ 9 & 2 & 1.6 & 98.4 \\ 10 & 2 & 1.6 & 100.0\end{array}$

${ }^{*} A$ continuous scale ranging from 1 to 10 was used to assess resondents' views on the feasibility of introducing the Mental Health Act 2001 at present levels of mental health service resourcing; with a score of 1 indicating 'completely infeasible'; 5 indicating 'moderately or partially feasible'; and 10 indicating 'completely feasible'.

lent resources. In addition, there have been several educational initiatives aimed at increasing professional and public awareness of the new legislation, organised by the Mental Health Commission, as well as various professional organisations, voluntary groups and service-user organisations.

Many of these organisations have also issued responses to the Mental Health Act 2001, some of which highlight the concerns also identified in this survey. A complete survey of responses to the Act is beyond the scope of this summary, but selected responses, commentaries and information are available from the Irish College of Psychiatrists (www.irishpsychiatry.com/comments.html), the Irish College of General Practitioners (www.icgp.ie), Schizophrenia Ireland (www.sirl.ie) and other groups.

In addition, particular concerns have been expressed about the implications of the Act for specific sectors within psychiatry, with, for example, the Faculty of Learning Disability Psychiatry of the Irish College of Psychiatrists expressing concern about issues related to consent to treatment and detention, in the context of intellectual disability. ${ }^{9}$ Further information on these issues, as well as issues related to definitions and the international context of recent developments, is available from the National Association for Intellectual Disability in Ireland (www.namhi.ie) and the Faculty of Learning Disability Psychiatry of the Irish College of Psychiatrists (www.irishpsychiatry.com/ld_public.htm) ${ }^{9}$

Some of the concerns identified in this survey (eg. problems with the transition to the new legislation) are likely to be addressed in greater detail in the regulations that will accompany the implementation of further stages of the Mental Health Act 2001. However, this survey indicates considerable concern about the impact that implementation may have on resources within the mental health sector (with, for example, $60.0 \%$ expecting full implementation to result in decreased levels of patient contact and over $80 \%$ expecting that implementation will decrease resources available for other areas of the mental health services). Concern has been expressed elsewhere at the overall levels and patterns of 

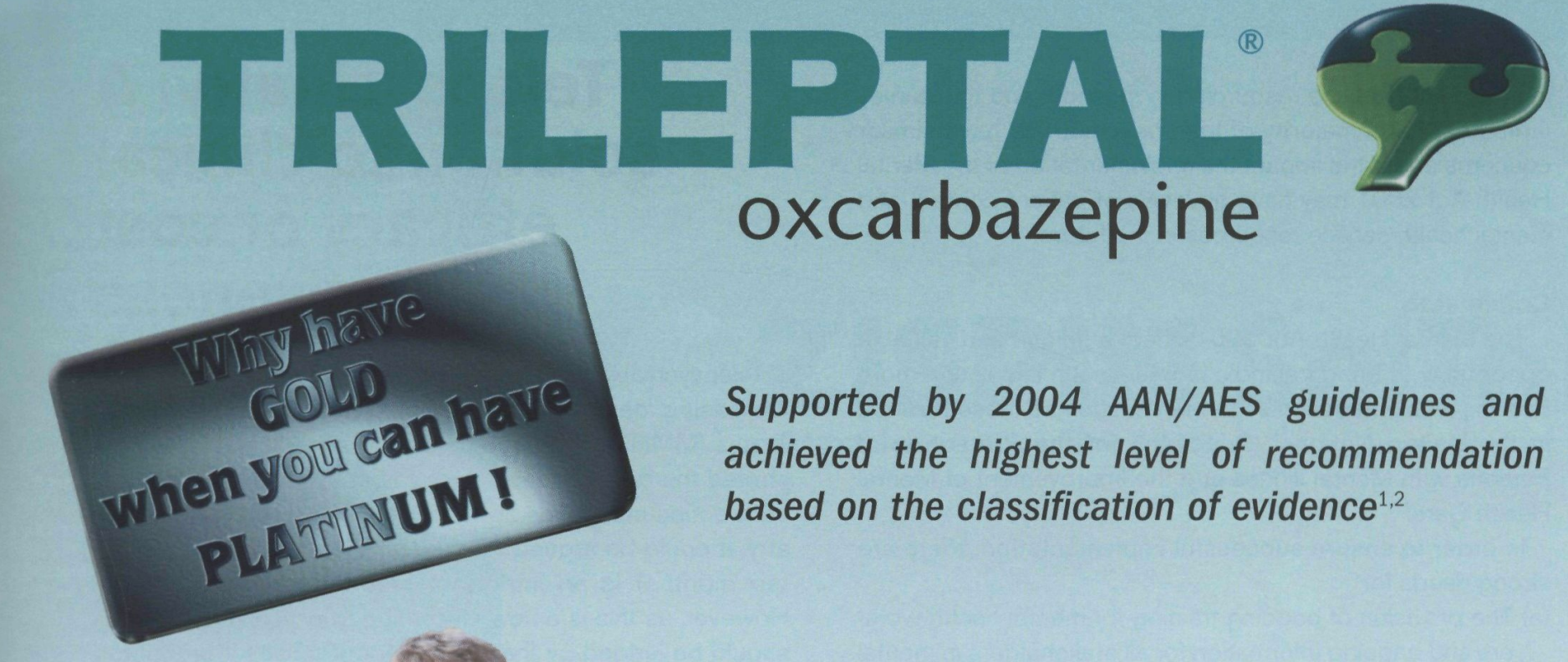

Supported by 2004 AAN/AES guidelines and achieved the highest level of recommendation based on the classification of evidence ${ }^{1,2}$

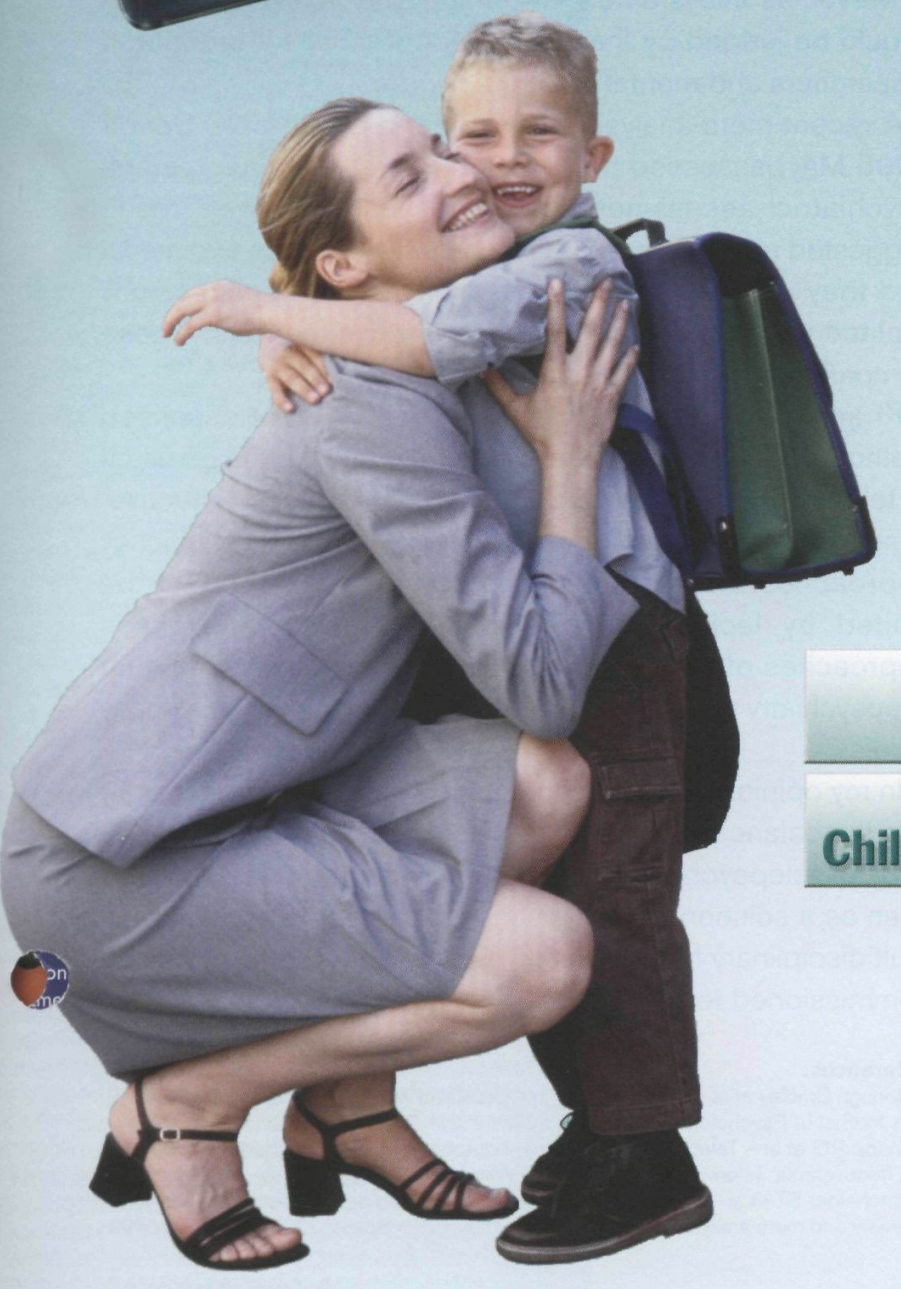

Established Efficacy and Safety ${ }^{4,5}$

Minimal Side Effects ${ }^{3.6}$

Trileptal $150 \mathrm{mg}, 300 \mathrm{mg}, 600 \mathrm{mg}$ Film-coated Tablets. Trileptal $60 \mathrm{mg} / \mathrm{ml}$ Oral Suspension

ABBREVIATED PRESCRIBING INFORMATION Refer to Summary of Product Characteristics (SmPC) before prescribing. PRESENTATION: Trileptal Filmcoated tablets containing $150 \mathrm{mg}$. $300 \mathrm{mg}$ or $600 \mathrm{mg}$ oxcarbazepine; Trileptal $60 \mathrm{mg} / \mathrm{ml}$ oral suspension containing $60 \mathrm{mg} / \mathrm{ml}$ oxcarbazepine. INDICATIONS: Epilepsy: partial seizures with or without generalized tonic-clonic seizures. DOSAGE AND ADMINISTRATION: Adults: 600 to $2,400 \mathrm{mg} /$ day. Children aged 6 years and above: 8 to 46

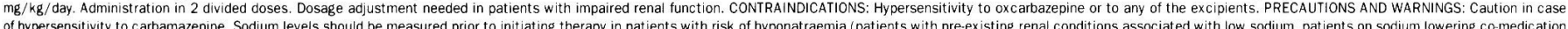
or NSAIDs) and levels should be monitored after 2 weeks and thereafter monthy for 3 months. Regular weight measurements in patients with cardiac insufficiency and sec condition, serum sodium should be checked). Caution in case of pre-existing conduction disturbances. Trileptal may render hormonal contraceptives ineffective. Caution in case of hepatitis. Caution when combined with alc ohol, when driving or operating machines. Use during pregnancy. the potential benefits should be carefully weighed asainst the potential risks to the foetus. Should not be used during breast.feeding. Trileptal oral suspension contains alcohol i $<100$ mg/dose), parabenes ( which may cause allergic reactions), and sorbitol (which should not be administered to patients with rare hereditary problems of fructose intolerance). Discontinuation of treatment should be done gradually. INTERACTIONS: Drugs metabolised by CYP2C19 (e.g. phenobarbitone, phenytoin), drugs metabolized by CYP3A4 and CYP3A5 (dihydropyridine calcium antagonists, oral contraceptives, and antiepileptic drugs). calcium antagonists. MAOIs, lithium. ADVERSE REACTIONS: Very Common ( $210 \%$ ): somnolence, headache, dizziness, diplopia, nausea, vomiting, fatigue. Common (21\%-<10\%); hyponatraemia, confusion, impaireo concentration, depression, apathy, agitation, aftect lability, ataxia, tremor, nystagmus, amnesia, vision disorders, vertigo. diarrhoea, constipation, abdominal pain, rash, alopecia, acne, asthenia. Uncommon ( $20.1 \%$ - <1\%): leucopenia, urticaria, increase in hepatic enzymes and alkaline phosphatase. Very rare (<0.01\%): thrombocytopenia, hypersensitivity, symptomatic hyponatraemia, arrhythmia, atrioventricular block, pancreatitis, and/or lipase and/or amylase increase, hepatitis, angioedema, Stevens-Johnson syndrome, toxic epidermal necrolysis (Lyell's syndrome), erythema multiforme, systemic lupus erythematosus, multi-organ hypersensitivity disorders. PACKAGE QUANTITIES: Trileptal Film-coated tablets $150 \mathrm{mg}$, $300 \mathrm{mg}, 600 \mathrm{mg}$ : Blister pack of 50 tablets; Trileptaloral suspension $60 \mathrm{mg} / \mathrm{ml}:$ Brown (amber) Type III glass bottle containing 250

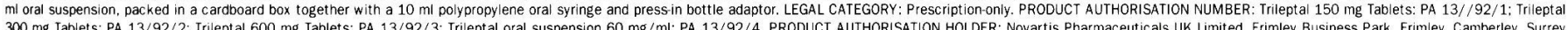
$300 \mathrm{mg}$ Tablets. PA $13 / 92 / 2$; Trleptal $600 \mathrm{mg}$ Tablets. PA $13 / 92 / 3$, Trieptal oral suspension $60 \mathrm{mg} / \mathrm{mll}$. PA $13 / 92 / 4$. PRODUCT AUTHORISATION HOLDER. Novartis Pharmaceutic als UK Limited, Frimley Business Park, Frimley, Camberley. Surrey GU16 7SA, UK. DAFE OF REVS ON OF THE TEXY: May 2005. Ful prescring

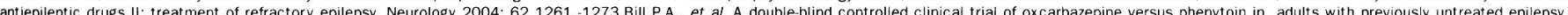
Epilepsy Research, 1997; 27: 195-204. 3. Summary of Product Characteristics Trileptal. 4. Bill P.A., et al. A double-blind controlled clinical trial of oxcarbazepine versus phenytoin in adults with previously untreated epilensy. Enilepsy Research. 1997: 27: 195.204. 5. Guerreiro M. M. et al. A double blind controlled clinica trial of oxcarbazepine versus phenytoin in children and adolescents with epilepsy. Enilepsy Research, 1997: 27: 205-213. 6. Barcs G. et al. Oxcarbazepine placebo-controlled, dose-ranging trial in refractory partial epilepsy. Epilepsia, 2000; 41(12): 1597-1607. 
mental health service resourcing in Ireland, ${ }^{10}$ and this survey indicates that a majority of Irish psychiatrists have similar concerns about the impact the implementation of the Mental Health Act 2001 may have on levels of patient contact and mental health service resources in the future.

\section{Conclusion}

The Mental Health Act 2001 offers a unique and valuable opportunity to bring Ireland's mental health legislation more into line with international standards, such as those outlined in the United Nations' 'Principles for the Protection of Persons with Mental IIIness and the Improvement of Mental Health Care."1

In order to ensure successful implementation, there are strong needs for

(a) The provision of ongoing training for mental health workers and ongoing information for all stakeholders in mental health services; and

(b) A careful re-consideration of the resources available for mental health services, in order to ensure both the effective implementation of this important legislation and the provision of an adequate standard of mental health care.

\section{Acknowledgements}

We are grateful to the Irish College of Psychiatrists for their assistance with this study. This paper does not necessarily represent the views of the Irish College of Psychiatrists. We also acknowledge the assistance of Ms Angela Robertson, Clinical Effectiveness Department, The State Hospital, Carstairs, Lanark, ML11 8R, UK, who advised on the design of the study.

\section{Brendan Kelly}

Consultant Psychiatrist and Senior Lecturer in Psychiatry

Department of Adult Psychiatry

UCD, Mater Misericordiae University Hospital Eccles St, Dublin 7, Ireland.

Fionnhar Lenihan

Specialist Registrar

The Carseview Centre, Medipark Dundee, Scotland DD2 1NH.

Declaration of interests: One of the authors of this paper (BDK) is trainee editor of the Irish Journal of Psychological Medicine.

\section{Telepsychiatry: A solution to clinical efficacy or cost effectiveness}

Telepsychiatry offers a promising means to reduce the ever increasing demands on Multidisciplinary Mental Health Teams (MMHT). The audit by Oonagh Bradley et al demonstrated the cost effectiveness of telepsychiatry and its user satisfaction rating was about $88.9 \%$ in favour of telepsychiatry. It could be argued that the short duration of the study (six months) is an important methodological limitation. However, as this is a new innovation to psychiatry, the audit should be judged by the excitng opportunities it brought to researchers and mental health providers.

A recent meta-analysis (Batchelder ST et al CNS Spectr 2005 May) assessed the replacement of certain in-person psychiatric assessment by telepsychiatry. Their result suggested no difference in efficacy between these two media and they postulated that telepsychiatry can successfully replace certain clinical and research situations. These comparisons are limited due to small effect sizes.

Knapp RG et al (Telemed JE Health 2004) assessed methodological issues of treatment outcomes in the research of telepsychiatry. In particular, they focus on cost effectiveness versus clinical efficacy. They advice that clinical efficacy approaches offer enhanced internal validity but may be limited by lack of generalisability. Cost effectiveness approaches offer more external validity. They suggest that telepsychiatry research should focus more on clinical efficacy studies.

In my opinion, future research should provide more insight into the balance between cost effectiveness and clinical efficacy of telepsychiatry. Finally, telepsychiatry should not be seen as a solution to the existing longstanding shortages of Multidisciplinary Mental Health Teams but rather it should be commissioned to compliment these teams.

\section{References:}

1.Oonagh Bradley et al - Telepsychiatry in a Child and Adolescent Psychiatric Services: Irish Journal of Psychological Medicine 2006: 23(1): 21-23.

2.Knapp RG et al - Telepsychiatry treatment outcome research methodology: Efficacy vs Effectiveness. Telemed JE Health 2004 Winter; 10 (4): 455-8.

3.Batchelder ST et al - Can telepsychiatry replace in-person psychiatric assessment? A review and meta-analysis of comparison studies. CNS Spectr 2005 May: 10(5): 40313

Adam Abba-Aji Registrar

Sligo Mental Health Service Journal Club Education Center, Sligo Mental Health Service Sligo Ireland.

\section{Correction}

Irish Journal of Psychological Medicine 2006; 23(1): 3-5, Neuropsychiatry of epilepsy. References should have run to 33 not 35 as in the text, a correction to the final proof was omitted. Full and correct text and references are available from niamh@medmedia.ie 